\section{Prevalência de fatores de risco cardiovascular em adolescentes}

\author{
Prevalence of cardiovascular risk factors in \\ adolescents
}

\begin{abstract}
The aim of this study was to determine the prevalence of cardiovascular risk factors in adolescents and to verify its association with age and gender. 644 high school students from public schools in the city of Londrina, Paraná State, Brazil, participated in the study. A two-step sampling process was used. Behavioral risk factors (physical inactivity, inadequate consumption of fruits and vegetables, and smoking) and biological risk factors (overweight and high blood pressure) were investigated. Nearly $90 \%$ of adolescents showed at least one risk factor. Inadequate consumption of fruits (56.7\%) and vegetables (43.9\%) and physical inactivity (39.2\%) were the most prevalent risk factors. Prevalence rates for high blood pressure and overweight were 18.6 and $12.7 \%$, respectively. Cardiovascular risk factors were more frequent among boys $(P R=1.20$; 95\%CI = 1.01-1.42). In conclusion, cardiovascular risk factors are a prevalent health issue among students in the city of Londrina.
\end{abstract}

Cardiovascular Diseases; Adolescent Health; Risk Factors

\author{
Marcelo Romanzini 1,2 \\ Felipe Fossati Reichert 1,3 \\ Adair da Silva Lopes 4 \\ Édio Luiz Petroski 4 \\ José Cazuza de Farias Júnior 2
}

\section{Introdução}

O processo de urbanização ocorrido em muitos países no último século acarretou modificações no estilo de vida da população (adoção de hábitos alimentares inadequados e inatividade física) que favoreceram o crescimento dos níveis de obesidade 1 . Atrelados a esse contexto, o processo de industrialização e o desenvolvimento tecnológico presentes na sociedade atual, contribuíram para uma alteração nas principais causas de mortalidade e morbidade, com predominância das doenças e agravos não transmissíveis (DANTs) em relação às doenças infecto-contagiosas e àquelas causadas por deficiência nutricional 2 .

Estimativas recentes apontaram que o grupo das DANTs responde por cerca de $60 \%$ da mortalidade ocorrida em todo o mundo, sendo as doenças cardiovasculares responsáveis por uma em cada três mortes registradas por este grupo de doenças ${ }^{3}$. Quadro semelhante foi observado no Brasil, onde a mortalidade por DANTs e por doenças cardiovasculares foi de $58 \%$ e $31,5 \%$, respectivamente (Sistema de Informações sobre Mortalidade. Indicadores e dados básicos - Brasil - 2004. http:// tabnet.datasus.gov.br/cgi/ tabcgi.exe?idb2004/c04.def, acessado em 12/ dez/2005).

A etiologia das DANTs, e, conseqüentemente das doenças cardiovasculares, tem origem na presença e/ou agrupamento de fatores de riscos inerentes ao próprio indivíduo (gerais, compor- 
tamentais e biológicos) ou à comunidade em que o mesmo se encontra inserido (condições sócio-econômicas, ambientais, culturais e de urbanização) ${ }^{4}$. Nesse sentido, a adolescência se caracteriza como um período propício para o desenvolvimento de estratégias intervencionistas voltadas ao combate das doenças cardiovasculares, uma vez que há evidências de que estas doenças podem se originar neste período de vida 5 . Além disso, diversos fatores de risco de origem biológica adquiridos na adolescência tendem a persistir até a maioridade 6,7, acentuando o risco de morbi-mortalidade na vida adulta 8,9.

A Organização Mundial da Saúde (OMS) 10 considera a escola como um ambiente apropriado para a implementação de programas de promoção da saúde, visando à prevenção de mortes prematuras e doenças. Todavia, estudos sobre fatores de risco cardiovascular em adolescentes e/ou escolares brasileiros são escassos 11,12,13,14. Sendo assim, o objetivo do presente estudo foi determinar a prevalência de fatores de risco cardiovascular de forma isolada e simultânea, em adolescentes do Ensino Médio diurno do Município de Londrina, Estado do Paraná.

\section{Materiais e métodos}

Entre julho e setembro de 2005, realizou-se um estudo transversal com escolares da rede pública de ensino do Município de Londrina. Indivíduos de ambos os sexos, matriculados em escolas da zona urbana e nas séries do Ensino Médio diurno foram elegíveis para o estudo.

O processo de amostragem foi realizado em dois estágios. Inicialmente, todas as escolas públicas foram listadas e agrupadas de acordo com sua localização geográfica (norte, sul, leste, oeste, centro e anel periférico). Seis escolas foram então selecionadas, adotando-se uma estratégia sistematizada, o que garantiu representatividade das zonas geográficas da cidade na amostra. O número de turmas selecionadas em cada escola foi definido de modo a alcançar a representatividade percentual de sua área geográfica em relação ao município como um todo. Por exemplo, se $20 \%$ de todas as turmas do município estão localizadas na região geográfica A, este foi o percentual de turmas desta região incluídas na amostra. A seleção das turmas em cada escola foi realizada por amostragem aleatória simples.

Para o cálculo do tamanho da amostra levou-se em consideração a menor prevalência esperada para os fatores de risco investigados (10\% para tabagismo) 15 , intervalo de $95 \%$ de confiança, erro de 3,0 pontos percentuais, efeito de delineamento de 1,5 e acréscimo de $20 \%$ para perdas/recusas. Com base nesses parâmetros o tamanho amostral mínimo seria de 669 sujeitos para participarem do estudo, sendo considerados elegíveis todos aqueles que se encontravam em sala de aula no dia da coleta. Os critérios de exclusão adotados foram: (a) idade superior a 19 anos; (b) presença de hipertensão secundária; (c) adolescentes grávidas; (d) indivíduos com ampla variação ( $\geq 4 \mathrm{mmHg}$ ) nos valores de pressão arterial sistólica e/ou diastólica no momento das aferições.

Os fatores de risco comportamentais foram avaliados por questionários elaborados com base em outros instrumentos previamente validados e/ou empregados em estudos com escolares.

O nível de atividade física total (atividades de lazer, domésticas, deslocamento e trabalho) foi estimado pela versão em Português do Questionário Internacional de Atividade Física (IPAQ), em sua versão curta (http://www.ipaq.ki.se/ ipaq.htm, acessado em 30/Abr/2005). Para tanto, computaram-se apenas os minutos de atividades físicas relacionadas aos esforços de intensidades moderadas e vigorosas. Foram considerados inativos os escolares com escore de atividade física inferior a 300 minutos/semana 16 .

O instrumento Youth Risk Behavior Survey 17 foi utilizado para a obtenção da freqüência mensal do consumo de cigarros. Assim, o tabagismo foi definido como o consumo de um ou mais cigarros nos últimos 30 dias. A freqüência do consumo semanal de frutas e verduras foi estimada utilizando-se o instrumento Global School-based Student Health Survey 18 . O consumo inadequado de frutas e verduras foi definido como uma ingestão inferior a quatro dias por semana. Esses instrumentos foram traduzidos e adaptados para a língua portuguesa, de modo a facilitar sua compreensão pelos adolescentes brasileiros, apresentando em uma subamostra $(\mathrm{n}=35)$, medidas de reprodutibilidade (kappa) variando entre 0,58 e 1,0.

Os fatores de risco biológicos investigados foram o excesso de peso corporal (sobrepeso e obesidade) e a pressão arterial elevada. Medidas antropométricas de massa corporal (kg) e estatura (m) foram coletadas por um único avaliador para o cálculo do índice de massa corporal (IMC). $\mathrm{O}$ excesso de peso corporal foi determinado de acordo com Cole et al. 19.

Aferições da pressão arterial sistólica (PAS) e diastólica (PAD) foram realizadas por meio do método auscultatório, mediante a utilização de um esfigmomanômetro do tipo aneróide, com manguitos de tamanhos apropriados à circunferência dos braços dos adolescentes, devidamente calibrados antes do início do período de 
avaliações. Duas mensurações com um intervalo mínimo de três minutos foram obtidas com os adolescentes sentados e em repouso por pelo menos cinco minutos. Foi padronizado que caso houvesse diferenças iguais ou maiores que $4 \mathrm{mmHg}$ entre as aferições, uma terceira mensuração seria realizada. Para fins de análise, considerou-se o valor médio das medidas. Todas as aferições foram realizadas por uma única avaliadora, devidamente familiarizada com a padronização adotada e com um elevado índice de reprodutibilidade (coeficiente intraclasse = 0,96 e 0,95 para PAS e PAD, respectivamente). Com base na referência do National High Blood Pressure Education Program dos Estados Unidos (NHBPEP) 20, os adolescentes com PAS e/ou PAD acima do percentil 90 para sexo, idade e estatura, ou aqueles com $\mathrm{PA} \geq 120 / 80 \mathrm{mmHg}$ foram considerados pré-hipertensos.

Anteriormente à análise, o banco de dados foi revisado e eventuais erros foram corrigidos. A razão de prevalência, mediante a utilização de regressão de Poisson, foi utilizada como medida de associação, com o intuito de analisar as possíveis associações existentes entre os fatores de risco investigados e as variáveis demográficas (sexo e idade). Adotou-se esse procedimento, ao invés da análise de regressão logística, em virtude de que a elevada prevalência de alguns fatores de risco (inatividade física e de hábitos alimentares inadequados) resultaria em superestimação dos valores de odds ratio quando comparados à razão de prevalência. As análises levaram em consideração o efeito de cluster da amostra, utilizando-se para tal o grupo de comandos "survey” do programa Stata 9.2 (Stata Corp., College Station, Estados Unidos). O nível de significância adotado foi de $5 \%$.

Este estudo foi aprovado pelo Comitê de Ética em Pesquisa com Seres Humanos da Universidade Federal de Santa Catarina (protocolo 096/05), de acordo com a Resolução no. 196/96 do Conselho Nacional de Saúde.

\section{Resultados}

Dos 719 adolescentes selecionados, 644 atenderam aos critérios de inclusão. Foram excluídos cinco escolares com idade superior a 19 anos, quatro devido a quadro de hipertensão secundária, três gestantes, vinte e dois devido a preenchimento incorreto do questionário, onze por apresentarem variações superiores a $4 \mathrm{mmHg}$ entre as aferições da pressão arterial sistólica e/ ou diastólica. Além disso, houve 30 adolescentes que se recusaram a participar do estudo.
A amostra foi predominantemente composta por moças $(61,6 \%)$. A média de idade foi de $16,4 \pm 1,0$ anos para os rapazes e de $16,2 \pm 1,0$ anos para as moças. A maioria dos jovens pertence aos níveis econômicos B $(52,6 \%)$ e C $(37,3 \%)$.

$\mathrm{O}$ consumo inadequado $(<4 \mathrm{dias} / \mathrm{semana})$ de frutas e verduras $(56,7 \%$ e $43,9 \%$, respectivamente) e a inatividade física $(39,2 \%)$ foram os comportamentos de risco mais freqüentes entre os adolescentes, enquanto apenas 5,1\% relataram consumir cigarros por pelo menos uma vez ao mês (Figura 1). A Tabela 1 apresenta a associação dos fatores de risco cardiovascular de origem comportamental com as variáveis sexo e idade.

Nenhum dos fatores de risco comportamentais foi associado às variáveis demográficas. A Tabela 2 apresenta a prevalência de excesso de peso corporal e de pressão arterial elevada entre os adolescentes investigados, em função do sexo e da idade.

Em relação à associação entre o sexo e o sobrepeso, os rapazes apresentaram maior propensão de possuírem excesso de peso ( $\mathrm{p}=$ $0,075)$. Associações significantes foram observadas entre a pressão arterial elevada e as variáveis demográficas. A probabilidade dos rapazes apresentarem valores elevados de pressão arterial é cerca de cinco vezes maior quando comparados às moças. No que se refere à idade, observou-se uma tendência linear de aumentos na prevalência de pressão arterial elevada ao longo das faixas etárias, com o maior risco sendo apresentado aos 18 anos.

A Tabela 3 apresenta a análise da simultaneidade dos fatores de risco (inatividade física, hábitos alimentares inadequados, tabagismo, excesso de peso corporal e pressão arterial elevada). Para tanto, em termos operacionais a identificação de hábitos alimentares inadequados foi considerada pela presença de um consumo semanal de frutas e/ou verduras inferior a 4 dias/semana.

Aproximadamente $35 \%$ dos adolescentes apresentaram dois ou mais fatores de risco comportamentais, enquanto cerca de $30 \%$ tinham pelo menos um fator de risco biológico. Considerando a presença de ambos os grupos (comportamentais e biológicos), observa-se que $45 \%$ dos adolescentes possuem dois ou mais fatores de risco e apenas 13,4\% não apresentam nenhum fator de risco.

A análise de associação entre as variáveis demográficas e a simultaneidade de fatores de risco demonstrou que, comparados às moças, os rapazes foram mais expostos à presença de pelo menos um fator de risco biológico. 


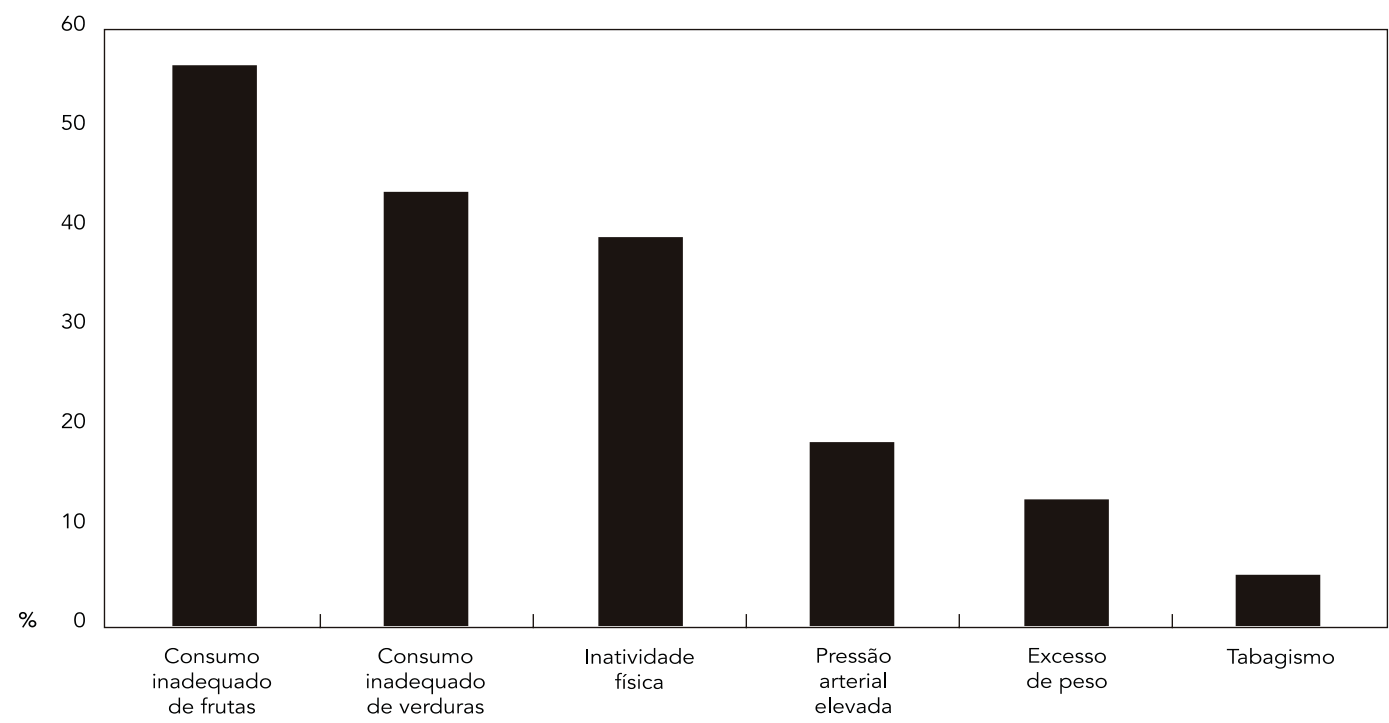

\section{Discussão}

Programas de promoção da saúde na escola têm sido preconizados por entidades internacionais 10 . Essa recomendação pode ser fundamentada pelo fato de que na adolescência são incorporados hábitos de vida que podem persistir até a fase adulta, além de que a presença de fatores de risco biológicos nesta fase da vida tem sido associada a um acentuado risco de morbidade $\mathrm{e}$ mortalidade na maioridade 8,9 .

O consumo inadequado de frutas e verduras, além da inatividade física, foram os fatores de risco mais prevalentes nos escolares da cidade de Londrina. Apesar das diferenças metodológicas quanto à instrumentação e critérios adotados, estudos nacionais também evidenciaram altas prevalências desses fatores de risco entre adolescentes 11,12,21. Considerando que a inatividade física e o consumo inadequado de frutas e verduras estão classificados entre os principais fatores de risco para morbi-mortalidade em adultos 22 , esse quadro torna-se preocupante, uma vez que os padrões alimentares e de atividade física adquiridos na adolescência tendem a permanecer até a vida adulta 23,24 .

Um aspecto comportamental positivo observado no estudo refere-se à baixa prevalência de tabagismo $(5,1 \%)$, a qual foi praticamente a me- tade daquela relatada por Galduróz et al. 15 em escolares dos ensinos fundamental e médio das redes municipal e pública das 27 capitais brasileiras $(9,9 \%)$. Todavia, deve-se considerar que a não inclusão de escolares pertencentes ao Ensino Médio noturno pode ter contribuído para a baixa prevalência de tabagismo encontrada no nosso trabalho.

A prevalência de pressão arterial elevada nos jovens investigados $(18,6 \%)$ foi superior àquelas verificadas em escolares das cidades de Maceió (Alagoas) 13 (7,7\%), e Belo Horizonte (Minas Gerais) 25 (10,2\%). Essa maior prevalência pode ser atribuída aos seguintes fatores: (a) o ponto de corte empregado no presente estudo para a caracterização da pressão arterial elevada $(\mathrm{P}>90)$ foi diferente daquele utilizado no levantamento de Maceió ( $\mathrm{P}>95)$ e; (b) o estudo de Belo Horizonte envolveu indivíduos mais jovens (entre 6 e 18 anos), o que contribuiu para a menor prevalência observada, visto que a pressão arterial elevada está positivamente associada com idade.

A identificação de associações entre variáveis demográficas e fatores de risco pode auxiliar no direcionamento de programas de intervenção. No presente estudo, a pressão arterial elevada foi associada às variáveis demográficas. A presença de níveis elevados de pressão arterial foi cerca 
Prevalência $(\mathrm{P})$ de fatores de risco comportamentais e razão de prevalência bruta (RP) com intervalo de 95\% de confiança (IC95\%), conforme as variáveis demográficas.



* Valor de $p$ do qui-quadrado;

** Valor do $\mathrm{p}$ de tendência linear.

Tabela 2

Prevalência (P) de fatores de risco biológicos e razão de prevalência bruta (RP) com intervalo de 95\% de confiança (IC95\%), conforme as variáveis demográficas.

\begin{tabular}{|c|c|c|c|c|c|c|}
\hline \multirow[t]{2}{*}{ Variável } & \multicolumn{3}{|c|}{ Excesso de peso } & \multicolumn{3}{|c|}{ Pressão arterial elevada } \\
\hline & $P(\%)$ & RP (IC95\%) & $\mathrm{p}$ & $P(\%)$ & RP (IC95\%) & $p$ \\
\hline Sexo * & & & 0,075 & & & $<0,001$ \\
\hline Feminino & 10,8 & 1 & & 7,3 & 1 & \\
\hline Masculino & 15,8 & $1,46(0,95-2,25)$ & & 36,8 & $5,04(3,68-6,91)$ & \\
\hline Idade (anos) ** & & & 0,925 & & & 0,002 \\
\hline 15 & 15,7 & 1 & & 10,7 & 1 & \\
\hline 16 & 11,3 & $0,72(0,58-0,89)$ & & 17,6 & $1,64(1,05-2,58)$ & \\
\hline 17 & 10,8 & $0,69(0,36-1,30)$ & & 18,5 & $1,71(0,71-4,16)$ & \\
\hline 18 & 15,3 & $0,98(0,43-2,22)$ & & 28,2 & $2,62(1,67-4,13)$ & \\
\hline
\end{tabular}

* Valor de p do qui-quadrado;

** Valor do $p$ de tendência linear.

de cinco vezes maior para o sexo masculino, e, 2,6 maior para os adolescentes com idade $\geq 18$ anos. A associação entre pressão arterial e o sexo masculino permaneceu praticamente inalterada mesmo após o ajuste para IMC, idade, nível de atividade física e consumo de frutas/verduras $(\mathrm{RP}=4,7$; IC95\% $=3,1-7,1)$. Sorof et al. 26 demonstraram que rapazes possuem maiores chances de serem hipertensos quando comparados às moças (razão de odds = 1,46; IC95\% = 1,31-1,64). Ro- sa et al., 27 observaram associação de magnitude superior àquela encontrada em nosso estudo, em que escolares do sexo masculino apresentaram quase nove vezes mais chances de possuírem níveis pressóricos elevados quando comparados às moças. Apesar de a pressão arterial ter sido aferida em condições idênticas para ambos os sexos no presente trabalho, a magnitude da associação observada para estas variáveis deve ser vista com cautela. 
Simultaneidade de fatores de risco comportamentais e biológicos em adolescentes.

\begin{tabular}{lccc}
\hline Variável & Masculino & $\begin{array}{c}\text { Prevalência (\%) } \\
\text { Feminino }\end{array}$ & Ambos os sexos \\
\hline $\begin{array}{l}\text { Comportamentais } \\
\text { Nenhum }\end{array}$ & 21,1 & 18,9 & 19,8 \\
1 fator & 49,6 & 47,1 & 48,1 \\
2 fatores & 28,0 & 32,2 & 30,6 \\
3 fatores & 1,2 & 1,8 & 1,6 \\
Biológicos & & & 72,0 \\
Nenhum & 53,8 & 83,4 & 24,5 \\
1 fator & 39,7 & 15,1 & 3,4 \\
2 fatores & 6,5 & 1,5 & 13,4 \\
Ambos & & & 41,1 \\
Nenhum & 10,6 & 15,1 & 33,9 \\
1 fator & 38,6 & 42,6 & 11,6 \\
2 fatores & 32,5 & 34,8 & 7,6 \\
$\quad$ ou mais fatores & 18,2 & &
\end{tabular}

Razão de prevalência (RP) bruta com intervalo de 95\% de confiança (IC95\%) para associação entre simultaneidade de fatores de risco e variáveis demográficas.

\begin{tabular}{|c|c|c|c|c|c|c|}
\hline \multirow[t]{3}{*}{ Variável } & \multicolumn{6}{|c|}{ Fatores de risco } \\
\hline & \multicolumn{2}{|c|}{ Comportamentais * } & \multicolumn{2}{|c|}{ Biológicos ** } & \multicolumn{2}{|c|}{ Ambos * } \\
\hline & RP (IC95\%) & $p$ & RP (IC95\%) & $p$ & RP (IC95\%) & $p$ \\
\hline Sexo $* \star \star$ & & 0,480 & & $<0,001$ & & 0,280 \\
\hline Feminino & 1 & & 1 & & 1 & \\
\hline Masculino & $0,86(0,52-1,43)$ & & $2,78(2,07-3,72)$ & & $1,20(0,81-1,77)$ & \\
\hline Idade (anos) \# & & 0,796 & & 0,085 & & 0,654 \\
\hline 15 & 1 & & 1 & & 1 & \\
\hline 16 & $0,72(0,45-1,15)$ & & $1,09(0,80-1,48)$ & & $0,80(0,66-0,96)$ & \\
\hline 17 & $0,87(0,67-1,13)$ & & $0,99(0,56-1,75)$ & & $0,88(0,68-1,14)$ & \\
\hline 18 & $0,87(0,63-1,21)$ & & $1,53(1,03-2,28)$ & & $1,02(0,78-1,35)$ & \\
\hline
\end{tabular}

$* 2$ fatores de risco;

$\star \star \geq 1$ fator de risco;

$\star \star \star$ Valor de $\mathrm{p}$ do qui-quadrado;

\# Valor do p de tendência linear.

A falta de associação entre a inatividade física e o sexo poderia ser considerada um resultado surpreendente, visto que a literatura tem evidenciado que as moças são menos ativas que os rapazes $11,14,21$. Todavia, o grau de clustering dos dados com relação à área geográfica contribuiu para esse resultado. Quando as análises foram realizadas sem considerar o efeito do delineamento, a associação foi significante $(R P=1,28$; IC95\% = 1,04-1,59).
O tabagismo e o excesso de peso não foram associados com as variáveis demográficas. De fato, a literatura tem evidenciado que o uso de cigarros entre adolescentes é similar em ambos os sexos 28,29. Por outro lado, não há consenso quanto à relação entre tabagismo e idade. Corroborando com o presente estudo, Farias Júnior \& Lopes 14 não verificaram associação entre o uso de tabaco e a idade, enquanto estudos de base populacional, realizados na cidade de Pelotas, 
observaram uma relação diretamente proporcional entre estas variáveis 28,29. Tais divergências podem ter ocorrido em virtude das características distintas das amostras envolvidas nessas pesquisas (base escolar e base populacional).

Comparados às moças, os rapazes apresentaram uma propensão de serem mais expostos ao excesso de peso corporal ( $p$ limítrofe de 0,075). Indícios apontam que essa relação pode alternar dependendo da região geográfica, sendo influenciada pelos aspectos ambientais e culturais peculiares a cada região. Analisando os dados referentes ao levantamento realizado pelo Instituto Brasileiro de Geografia e Estatística (IBGE) entre os anos de 1996-1997 nas regiões Sudeste e Nordeste, Magalhães \& Mendonça 30 verificaram que o risco de exposição ao excesso de peso foi notável em moças nordestinas, ao passo que, na região Sudeste, as moças apresentaram menor risco de possuírem excesso de peso quando comparadas aos rapazes. Assim, dada a proximidade geográfica entre a cidade onde foi realizada a presente pesquisa e a Região Sudeste, infere-se que a maior prevalência de excesso de peso corporal notada entre os rapazes está em conformidade com a literatura nacional.

Embora o monitoramento da prevalência dos fatores de risco cardiovascular de maneira isolada possa contribuir para o planejamento e direcionamento de estratégias intervencionistas, a identificação da proporção de indivíduos que apresentam agregação de fatores de risco tornase extremamente importante, dado que as doenças crônicas não transmissíveis (DCNTs) são multicausais ${ }^{4}$. No presente estudo, a análise de simultaneidade demonstrou que aproximadamente $50 \%$ dos adolescentes apresentam dois ou mais fatores de risco cardiovasculares. É válido ressaltar que esse quadro pode ser ainda mais desfavorável, uma vez que não foram incluídas informações quanto à presença de outros fatores de risco de ordem biológica, tais como a hipercolesterolemia e o diabetes.

A presença simultânea de fatores de risco cardiovasculares ( $\geq 2$ fatores de risco) não foi associada às variáveis demográficas (Tabela 4). Contudo, quando as análises consideraram a natureza dos fatores de risco, associações foram observadas apenas para os fatores de risco biológicos, com os rapazes apresentando uma probabilidade 2,7 vezes maior de possuírem pelo menos um fator de risco. Esse quadro pode ser desfavorável à saúde masculina na fase adulta, tendo em vista que fatores de risco de origem biológica presentes na adolescência tendem a persistir até a vida adulta 6,7 , acentuando o risco de morbi-mortalidade por doenças cardiovasculares 8,9.

A escassez de estudos na literatura que analisaram a agregação de fatores de risco biológicos e comportamentais de forma conjunta dificulta a comparação dos nossos achados. Em uma revisão da literatura, Guerra et al. 31 constataram que ainda não existe um consenso em relação ao sexo que apresenta maior simultaneidade dos fatores de risco de natureza biológica. Aparentemente, essa relação parece ser influenciada pelos pontos de corte utilizados para a caracterização da presença dos respectivos fatores de risco 32 .

Algumas limitações do presente trabalho devem ser observadas. Os resultados do estudo podem não ser integralmente extrapolados para os adolescentes da cidade de Londrina, uma vez que o estudo foi realizado apenas em escolas públicas e com escolares das séries diurnas do Ensino médio. Nesse sentido, é plausível sugerir que as prevalências dos fatores de risco encontradas no presente estejam subestimadas pela inelegibilidade de estudantes do noturno ou mesmo adolescentes não-escolares. Outra limitação se refere à utilização da freqüência do consumo de frutas e verduras como indicador de hábito alimentar associado ao risco cardiovascular. Entretanto, essa medida também vem sendo empregada em outros estudos sobre fatores de risco em adolescentes 33,34 .

Em conclusão, os dados apresentados neste trabalho indicam que os fatores de risco cardiovascular constituem um problema de prevalência elevada em escolares do Ensino Médio diurno do Município de Londrina. Além disso, escolares do sexo masculino apresentaram maior agregação de fatores de risco biológicos. Nossos achados reforçam a necessidade da implementação de programas de saúde em ambiente escolar e podem ser utilizados como linha de base para futuros estudos. 


\section{Resumo}

O objetivo do trabalho foi determinar a prevalência de fatores de risco cardiovasculares biológicos e comportamentais em adolescentes e verificar sua associação com idade e sexo. Participaram do estudo 644 escolares da rede pública de ensino de Londrina, Paraná, Brasil. A seleção da amostra foi realizada em dois estágios. Fatores de risco comportamentais (inatividade física, consumo inadequado de frutas e verduras, e tabagismo) e biológicos (excesso de peso corporal e pressão arterial elevada) foram investigados. Quase $90 \%$ dos adolescentes apresentaram pelo menos um fator de risco. O consumo inadequado de frutas $(56,7 \%) e$ verduras $(43,9 \%)$ e, a inatividade física $(39,2 \%)$ foram os fatores de risco mais prevalentes. A prevalência de pressão arterial alta e excesso de peso foram de 18,6 e 12,7\%, respectivamente. Os fatores de risco cardiovascular foram mais prevalentes entre os rapazes (RP = 1,20; IC95\%: 1,01-1,42). Concluiu-se que os fatores de risco cardiovascular representam um problema de saúde com alta prevalência entre os escolares de Londrina.

Doenças Cardiovasculares; Saúde do Adolescente; Fatores de Risco

\section{Referências}

1. Popkin BM. Urbanization, lifestyle changes and the nutrition transition. World Development 1999; 27:1905-16.

2. Yusuf S, Reddy S, Ôunpuu S, Anand S. Global burden of cardiovascular diseases. Part I: general considerations, the epidemiologic transition, risk factors, and impact of urbanization. Circulation 2001; 104:2746-53.

3. World Health Organization. Changing history. http://whqlibdoc.who.int/whr/2004/924156265X. pdf (acessado em 12/Dez/2005).

4. Pan American Health Organization. CARMEN - conjunto de acciones para la reducción multifactorial de las enfermedades no transmisibles. http://www.paho.org/english/ad/dpc/nc/ carmen-info.htm (acessado em 01/Jun/2007).

5. Berenson GS, Srinivasan SR, Bao W, Newman 3rd WP, Tracy RE, Wattigney WA. Association between multiple cardiovascular risk factors and atherosclerosis in children and young adults. N Engl J Med 1998; 338:1650-6.

6. Bao W, Threefoot SA, Srinivasan SR, Berenson GS. Essential hypertension predicted by tracking of elevated blood pressure from childhood to adulthood: the Bogalusa Heart Study. Am J Hypertens 1995; 8:657-65.

\section{Colaboradores}

M. Romanzini, F. F. Reichert e J. C. Farias Júnior realizaram as análises e redigiram a primeira versão do manuscrito. A. S. Lopes e E. L. Petroski revisaram e incluíram modificações à versão final do manuscrito.

\section{Agradecimentos}

À Coordenação de Aperfeiçoamento de Pessoal de Nível Superior (CAPES), pela bolsa outorgada para a execução do estudo. Ferdinando Oliveira Carvalho e Durcelina Schiavoni, pelo auxílio prestado nas coletas de dados.
7. Freedman DS, Khan LK, Serdula MK, Dietz WH, Srinivasan SR, Berenson GS. The relation of childhood BMI to adult adiposity: the Bogalusa Heart Study. Pediatrics 2005; 115:22-7.

8. Engeland A, Bjorge T, Sogaard AJ, Tverdal A. Body mass index in adolescence in relation to total mortality: 32-year follow-up of 227,000 Norwegian boys and girls. Am J Epidemiol 2003; 157:517-23.

9. Raitakari OT, Juonala M, Kähönen M, Taittonen L, Laitinen T, Mäki-Torkko N, et al. Cardiovascular risk factors in childhood and carotid artery intima-media thickness in adulthood: the Cardiovascular Risk in Young Finns Study. JAMA 2003; 290:2277-83

10. World Health Organization. School health and youth health promotion. http://www.who.int/ school_youth_health/en/ (acessado em 15/ Set/2007).

11. Farias Júnior JC, Mendes JKF, Barbosa DBM. Associação entre comportamentos de risco à saúde em adolescentes. Rev Bras Cineantropom Desempenho Hum 2007; 9:250-6. 
12. Ribeiro RQC, Lotufo PA, Lamounier JA, Oliveira RG, Soares JF, Botter DA. Fatores adicionais de risco cardiovascular associados ao excesso de peso em crianças e adolescentes. O estudo do coração de Belo Horizonte. Arq Bras Cardiol 2006; 86: 408-18.

13. Silva MAM, Rivera IR, Ferraz MRMT, Pinheiro AJT, Alves SWS, Moura AA, et al. Prevalência de fatores de risco cardiovascular em crianças e adolescentes da rede de ensino da Cidade de Maceió. Arq Bras Cardiol 2005; 84:387-92.

14. Farias Júnior JC, Lopes AS. Comportamentos de risco relacionados à saúde em adolescentes. Rev Bras Ciênc Mov 2004; 12:7-12.

15. Galduróz JCF, Noto AR, Fonseca AM, Carlini EA. V levantamento nacional sobre o consumo de drogas psicotrópicas entre estudantes do ensino fundamental e médio da rede pública de ensino nas 27 capitais brasileiras 2004. São Paulo: CLR Balieiro Editores; 2005.

16. Biddle S, Cavill N, Sallis J. Young and active? Young people and health-enhancing physical activity evidence and implications. London: Health Education Authority; 1998.

17. Centers for Disease and Control. 2005 state and local youth risk behavior survey. http:// www.cdc.gov/HealthyYouth/yrbs/pdfs / 2005highschoolquestionnaire.pdf (acessado em 30/Abr/2005).

18. World Health Organization. Global schoolbased student health survey. http://www.who. int/school_youth_health/media/en/gshs_chile_ questionnaire2004.pdf (acessado em 30/ Abr/2005).

19. Cole TJ, Bellizzi MC, Flegal KM, Dietz WH. Establishing a standard definition for child overweight and obesity worldwide: international survey. BMJ 2000; 320:1240-3.

20. National High Blood Pressure Education Program Working Group on High Blood Pressure in Children and Adolescents. The fourth report on the diagnosis, evaluation, and treatment of high blood pressure in children and adolescents. Pediatrics 2004; 114:555-76.

21. Oehlschlaeger MH, Pinheiro RT, Horta B, Gelatti C, San'Tana P. Prevalência e fatores associados ao sedentarismo em adolescentes da área urbana. Rev Saúde Pública 2004; 38:157-63.

22. World Health Organization. The world health report 2002 - reducing risks, promoting healthy life. http://www.who.int/whr/2002/en/whr02_en.pdf (acessado em 27/Nov/2007).
23. Lake AA, Mathers JC, Rugg-Gunn AJ, Adamson AJ. Longitudinal change in food habits between adolescence (11-12 years) and adulthood (32-33 years): the ASH30 Study. J Public Health 2006; 28:10-6.

24. Azevedo MR, Araujo CL, Silva MC, Hallal PC. Tracking of physical activity from adolescence to adulthood: a population-based study. Rev Saúde Pública 2007; 41:69-75.

25. Oliveira RG, Lamounier JA, Oliveira ADB, Castro MDR, Oliveira JS. Pressão arterial em escolares e adolescentes - o estudo de Belo Horizonte. J Pediatr (Rio J) 1999; 75:256-66.

26. Sorof JM, Lai D, Turner J, Poffenbarger T, Portman RJ. Children overweight, ethnicity, and the prevalence of hypertension in school-aged. Pediatrics 2004; 113:475-82.

27. Rosa MLG, Fonseca VM, Oigman G, Mesquita ET. Pré-hipertensão arterial e pressão de pulso aumentada em adolescentes: prevalência e fatores associados. Arq Bras Cardiol 2006; 87:46-53.

28. Malcon MC, Menezes AMB, Chatkin M. Prevalência e fatores de risco para tabagismo em adolescentes. Rev Saúde Pública 2003; 37:1-7.

29. Horta BL, Calheiros P, Pinheiro RT, Tomasi E, Amaral KC. Tabagismo em adolescentes de área urbana na região Sul do Brasil. Rev Saúde Pública 2001; 35:159-64.

30. Magalhães VC, Mendonça GAS. Prevalência e fatores associados a sobrepeso e obesidade em adolescentes de 15 a 19 anos das regiões Nordeste e Sudeste do Brasil, 1996 a 1997. Cad Saúde Pública 2003; 19 Suppl 1:S129-39.

31. Guerra S, Oliveira J, Ribeiro JC, Teixeira-Pinto A, Duarte JA, Mota J. Relação entre a atividade física regular e a agregação de fatores de risco biológicos das doenças cardiovasculares em crianças e adolescentes. Rev Bras Saúde Matern Infant 2003. 3:9-15.

32. Goodman E, Daniels SR, Morrison JA, Huang B, Dolan LM. Contrasting prevalence of and demographic disparities in the World Health Organization and National Cholesterol Education Program Adult Treatment Panel III definitions of metabolic syndrome among adolescents. J Pediatr 2004; 145:445-51.

33. Baș M, Altan T, Dinçer D, Aran E, Kaya HG, Yüksek O. Determination of dietary habits as a risk factor of cardiovascular heart disease in Turkish adolescents. Eur J Nutr 2005; 44:174-82.34. Pileggi C, Carvone V, Nobile CGA, Pavia M. Blood pressure and related cardiovascular disease risk factors in 6-18 year-old students in Italy. J Paediatr Child Health 2005; 41:347-52.

Recebido em 06/Dez/2007

Versão final reapresentada em 01/Abr/2008

Aprovado em 16/Abr/2008 